\title{
Clinical significance of a single nucleotide polymorphism and allelic imbalance of matrix metalloproteinase-1 promoter region in prostate cancer
}

\author{
NORIHIKO TSUCHIYA ${ }^{1}$, SHINTARO NARITA ${ }^{1}$, TERUAKI KUMAZAWA ${ }^{1}$, TAKAMITSU INOUE ${ }^{1}$, \\ ZHIYONG MA ${ }^{1}$, HIROSHI TSURUTA ${ }^{1}$, MITSURU SAITO ${ }^{1}$, YOHEI HORIKAWA ${ }^{1}$, \\ TAKESHI YUASA $^{1}$, SHIGERU SATOH ${ }^{1}$, OSAMU OGAWA ${ }^{2}$ and TOMONORI HABUCHI ${ }^{1}$ \\ ${ }^{1}$ Department of Urology, Akita University School of Medicine, 1-1-1 Hondo Akita 010-8543; \\ ${ }^{2}$ Department of Urology, Graduate School of Medicine, Kyoto University, \\ 54 Shogoin, Kawahara-cho, Sakyo-ku, Kyoto 606-8507, Japan
}

Received April 16, 2009; Accepted June 5, 2009

DOI: $10.3892 /$ or_00000462

\begin{abstract}
Matrix metalloproteinase-1 (MMP-1) is associated with cancer invasion and metastasis. The $2 G$ allele of the polymorphic site in the $M M P-1$ promoter was demonstrated to have a higher transcription activity than the $1 G$ allele. Allelic imbalance (AI) at $11 \mathrm{q} 22$ harboring the $M M P-1$ is frequently observed in various cancers and may be associated with an advanced disease. We conducted a case-control study to determine the association of the $M M P-1$ genotype with susceptibility to prostate cancer involving 283 prostate cancer patients and 251 controls. Furthermore, AI, retention allele of the $M M P-1$ promoter, and MMP-1 protein expression were analyzed in 77 prostate cancer specimens. The $M M P-1$ promoter polymorphism was associated with neither susceptibility nor progression of prostate cancer. Tumors with $1 G / 2 G$ and $2 G / 2 G$ genotypes had a significantly higher MMP-1 expression level compared to those with $1 G / 1 G$ genotype ( $\mathrm{P}=0.006$ and 0.013 , respectively). $\mathrm{AI}$ at $11 \mathrm{q} 22$ was observed in $13(40.6 \%)$ of 32 informative cases. Retention of the $1 G$ and $2 G$ alleles were observed in 4 and 9 cases, respectively. AI was significantly associated with the Gleason score $(\mathrm{P}=0.003)$ and pathological stage $(\mathrm{P}=0.022)$. In addition, retention of the $2 G$ allele showed a significant association with the pathological stage $(\mathrm{P}=0.026)$. AI at $11 \mathrm{q} 22$ region, retention of the $2 G$ allele, specifically appeared to be involved in the progression of prostate cancer. However, the presence of the $2 G$ allele of the $M M P-1$ promoter polymorphism itself seems to influence neither the susceptibility nor the progression of prostate cancer.
\end{abstract}

Correspondence to: Dr Norihiko Tsuchiya, Department of Urology, Akita University School of Medicine, 1-1-1 Hondo Akita 010-8543, Japan

E-mail address: tsuchiya@med.akita-u.ac.jp

Key words: prostate cancer, matrix metalloproteinase-1, polymorphis, allelic imbalance

\section{Introduction}

Tumor invasion or metastasis is one of the most crucial events for determining the outcome of cancer patients. In the first step of these events, several proteinases such as serine proteinase urokinase plasminogen activator and matrix metalloproteinases (MMPs) degrade the extra-cellular matrix (ECM), so that the tumor cells can migrate to the stromal tissue through the basement membrane (1). MMP-1, a member of MMP family, is abundantly expressed in many types of cancer cells and adjacent stromal fibroblasts (2-5), and digests various elements of the ECM including collagen types I, II, II, VII, VIII, X, and XI (6). The overexpression of MMP-1 has been shown to be associated with tumor progression and poor outcomes for cancers of the digestive system and melanoma $(3,5,7,8)$.

The expression of $M M P-1$ is mainly regulated by activated protein-1 (AP-1) transcription factor that mediates signal transduction from cytokines and growth factors such as interferons, interleukins, epidermal growth factor, and fibroblast growth factor (9). In the promoter region of the $M M P-1$, three AP-1 binding sites are located at $-72,-186$, and $-1062 \mathrm{bp}$ from the transcription start site. Insertion of an extra guanine residue adjacent to the AP-1 binding site at -1062 bp creates a new binding site for ETS transcription factor at -1602 bp $\left(5^{\prime}-A A \underline{G G A T-3}\right.$ '; $\left.2 G\right)$, and both the AP-1 and ETS cooperatively enhance the expression of $M M P-1$ (10). AP-1 binding to the -1062 bp acts as a repressor of transcription when the polymorphic site has only one guanine residue $\left(5^{\prime}-A A \underline{G} A T-3\right.$ '; $\left.1 G\right)$ (11).

Recent epidemiological studies have demonstrated almost consistent results regarding the biological function of the $M M P-1$ promoter polymorphism and its association with the susceptibility or progression in several cancer types (4,12-23). The $2 G$ allele of the polymorphism has been reported to be related with increased risk of colorectal, lung, endometrial, ovary, kidney, and bladder cancers $(4,12-20)$, while the $2 G$ allele was associated with tumor progression or patient survival but not with susceptibility of uterus, stomach, and colorectal 
cancers (21-23). However, the results of most studies are consistent with each other regarding the risk associated with the $2 G$ allele. Meanwhile, $11 \mathrm{q} 22$ harboring the $M M P-1$ gene exhibits amplification in esophageal, colorectal, cervical, and prostate cancers (24-27). Determining which of the polymorphic alleles is amplified and how the amplified allele affects the expression of MMP-1 or phenotypes of cancers is also of interest. In prostate cancer, immunohistochemical and in situ hybridization analysis have detected MMP-1 in cancer cells as well as in normal adjacent and prostatic intraepithelial cells (28). However, a previous study that evaluated the effect of the polymorphism on prostate cancer found no association between MMP-1 and susceptibility or metastatic status of prostate cancer (29). There have been few studies focusing on the clinical implication of MMP-1 expression, promoter polymorphism, and allelic imbalance (AI) of 11q22.

We hypothesized that the $1 G / 2 G$ polymorphism or AI of the $M M P-1$ promoter region affects the development and/or progression of prostate cancer. To validate this hypothesis, we first conducted a case-controlled study to examine the effect of the polymorphism on the risk and progression of prostate cancer, and then investigated how the genotype and AI of the region affected the malignant potential, invasiveness of the tumors, and protein expression of MMP-1.

\section{Materials and methods}

Subjects. We studied a series of 524 registered subjects, including 283 patients with prostate cancer and 251 control males at the Akita University Medical Center and related community hospitals in Akita prefecture, who agreed to participate in this study and provided blood specimens. Prostate cancer patients were selected from April 1997 to December 2003 and control subjects were selected from March 1998 to September 2001.

For all patients with prostate cancer, histological evaluation was performed on specimen obtained by transrectal needle biopsy or transurethral resection of the prostate for voiding symptoms. The clinical or pathological stage of prostate cancer at the time of diagnosis was determined by reviewing the medical records based on the Tumor-Node-Metastasis system. Prostate cancer was classified into stage A (T1a-bNOM0), stage B (T1c-2N0M0), stage C (T3-4NOM0), or stage D (T14N1M0-1 or T1-4N0-1M1) by the modified Whitmore-Jewett system. In patients who underwent radical prostatectomy, the final pathological stage was applied and in patients who did not undergo radical prostatectomy, clinical stage was applied. Pathological grading of PCa was determined according to the General Rule for Clinical and Pathological Studies on Prostate Cancer by the Japanese Urological Association and the Japanese Society of Pathology, which is mostly based on the WHO criteria and the Gleason score. All pathological grading was based on needle biopsy specimens in stages B-D patients and surgical specimens in stage A patients. Well, moderately, and poorly differentiated carcinomas generally correspond to Gleason scores of 2-4, 5-7, and 8-10, respectively. In the present study, because the two grading systems were individually used by local pathologists, the tumor grading system was newly categorized as follows: low grade cancer included well-differentiated or Gleason 2-4 carcinomas, inter- mediate grade cancer included moderately differentiated or Gleason 5-7 carcinomas, and high grade cancer included poorly differentiated or Gleason 8-10 carcinomas. No definitive pathological grade could be determined in 24 patients due to inadequate information or inappropriate classification by local pathologists.

The male controls comprised 251 volunteers without any apparent voiding symptoms. They were selected randomly from the native Japanese population attending communitybased medical check-ups. They were all tested for total serum PSA levels (the Tandem-R assay), and those with abnormal total PSA levels $(4.0 \mathrm{ng} / \mathrm{ml}$ or more) were omitted from the study. Written informed consent was obtained from all the subjects. The present study was approved by the Institutional Review Board of the Akita University School of Medicine, Akita, Japan.

Genotyping of the MMP-1 promoter polymorphism. DNA was extracted from the collected blood samples of each subject using a QIAamp Blood Kit (Qiagen, Hilden, Germany). The PCR was performed in a final volume of $15 \mu 1$ containing 20 ng genomic DNA, 5 pmol forward primer (5'-GTT ATG CCA CTT AGA TGA GG-3'), 5 pmol reverse primer (5'-CTT GGA TTG ATT TGA GAT AAG-3'), 0.2 mM of each dNTP (dATP, dCTP, dGTP, and dTTP), $1.0 \mathrm{mM} \mathrm{MgCl}_{2}$, and 0.5 unit of Ampli-Taq Gold DNA polymerase (Perkin-Elmer, Branchburg, NJ). Initial denaturation at $94^{\circ} \mathrm{C}^{\circ} \mathrm{C}$ for $30 \mathrm{sec}$, annealing at $55^{\circ} \mathrm{C}$, and extension at $72^{\circ} \mathrm{C}$ for $30 \mathrm{sec}$ with final extension at $72^{\circ} \mathrm{C}$ for $7 \mathrm{~min}$. The forward primer was labeled with a fluorescent dye, HEX, to examine the size of PCR products with an autosequencer (ABI 310, Applied Biosystems, Foster, CA) as described previously (30). The size of the PCR products was determined in comparison with an internal ROX 400-size standard (Applied Biosystems) and analyzed using GENESCAN software v3.1 (Applied Biosystems) (Fig. 1A). The 72-bp and 73-bp fragments of amplified DNA are equivalent to $1 G$ allele and $2 G$ allele, respectively. The validity of the analysis was confirmed by direct sequencing of several PCR samples using ABI PRISM 310 DNA sequencer (Applied Biosystems).

Analysis of allelic imbalance (AI). AI of the polymorphic site of $M M P-1$ promoter was analyzed using DNA pairs obtained from the tumor and peripheral blood samples in a subgroup of 77 patients who underwent radical prostatectomy. Pathological diagnosis was confirmed by microscopic examination of hematoxylin and eosin (H\&E)-stained sections. Regions of $>80 \%$ tumor density were marked on H\&E-stained slides to be used as guidelines for microdissection. The target foci were microdissected using a 20-gauge needle, comparing the slide with the H\&E staining in the same position. DNA was extracted using a DEXPAT kit (Takara Biomedical Inc., Shiga, Japan), precipitated in cold ethanol with sodium acetate and Pellet Paint NF, Co-Precipitant (Novagen, Madison, WI), and resuspended in Tris-EDTA buffer. Peripheral blood and corresponding prostate tissue DNA samples were simultaneously amplified and both the PCR products were analyzed using ABI PRISM 310 DNA sequencer as described above. AI was determined by measuring the signal imbalance between the opposing alleles. Presence of AI was detected when one 
A

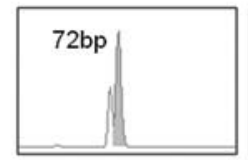

$1 G / 1 G$

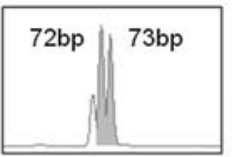

$1 G / 2 G$

B

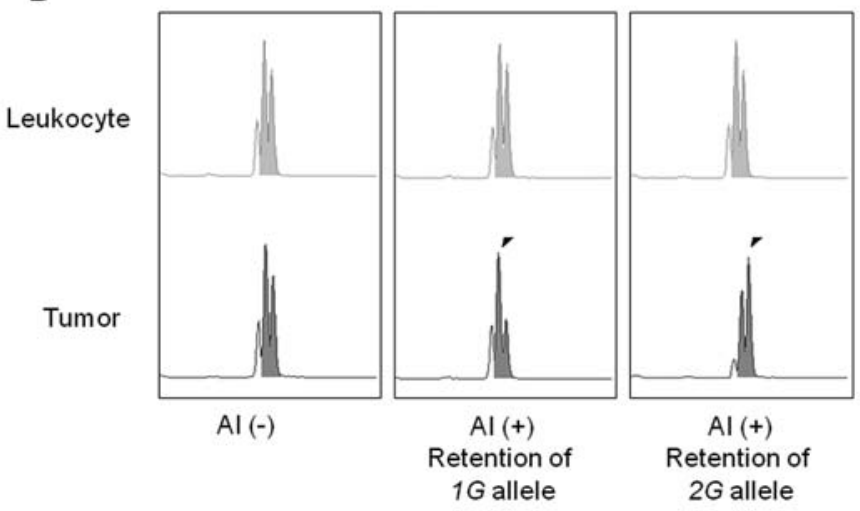

Figure 1. Genotyping the $M M P-1$ promoter insertion polymorphism using autosequencer and GENESCAN software are illustrated (A). $1 G$ and $2 G$ alleles are identified as 72-bp and 73-bp PCR amplified DNA fragments, respectively. Allelic imbalance (AI) is determined when one of the two signal peaks in a PCR product from the prostate tumor DNA was $<70 \%$ of that from the corresponding peripheral blood (leukocyte) DNA. Representative results for AI analysis, no AI, retention of the $1 G$ allele, and retention of the $2 G$ allele (from the left to the right), are shown (B). Arrows indicate retention alleles.

of the signal peaks in a PCR product from prostate tumor DNA was $<70 \%$ of that from the corresponding peripheral blood DNA on the GENESCAN software (Fig. 1B). Although the AI was reportedly attributed to amplifications of the $11 \mathrm{q} 22$ region containing the $M M P-1$ promoter, an allele that had a significantly higher signal peak was determined as a retained allele because the absolute copy number of the gene was not determined in this study.

Immunohistochemical analysis of MMP-1 expression. Seventy-seven prostate specimens obtained at radical prostatectomy were subject to immunohistochemical analysis. The specimens were fixed in $10 \%$ buffered formalin and embedded in paraffin. Individual paraffin blocks containing cancer lesions with representative Gleason scores were selected from each specimen. Tissue sections $(5 \mu \mathrm{m})$ were de-paraffinized in xylene and rehydrated through a graded ethanol series. Endogenous peroxidase activity was blocked with $0.3 \%$ hydrogen peroxide for $15 \mathrm{~min}$. The sections were boiled in $0.01 \mathrm{M}$ citric acid ( $\mathrm{pH} \mathrm{6.0)}$ to retrieve the antigen and non-specific binding was blocked with $5 \%$ goat serum for $10 \mathrm{~min}$. After washing, mouse monoclonal anti-human MMP1 antibody (F-67; Daiichi Fine Chemical Co., Ltd., Toyota, Japan) at 1:1000 was applied and incubated at $4^{\circ} \mathrm{C}$ overnight. After washing in PBS, secondary antibody conjugated with horseradish peroxidase (EnVIsion system; Dako, Japan Inc., Tokyo, Japan) was applied, followed by incubation at room temperature for $30 \mathrm{~min}$. After washing in PBS again, tissue sections were developed with diaminobenzidine
Table I. Demographic data of subjects analyzed in the cohort study.

\begin{tabular}{lrc}
\hline & Cases & Controls \\
\hline Total number & 283 & 251 \\
Mean age (years \pm SD) & $72.0 \pm 8.3$ & $70.0 \pm 7.6$ \\
Tumor stage & & \\
A & 24 & \\
B & 101 & \\
C & 49 & \\
D & 109 & \\
Tumor grade & & \\
Low & 49 & \\
Intermediate & 114 \\
High & 96 \\
Unknown & 24 \\
\hline
\end{tabular}

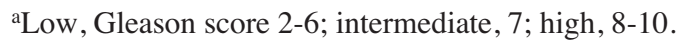

(DAB; Nichirei Biosciences Inc., Tokyo, Japan) and counterstained with hematoxylin.

The expression of MMP-1 was classified into four categories according to the staining intensity (i.e., negative, weak, intermediate, and strong) (Fig. 2). Assessment was performed by two independent observers unaware of clinical information. In the statistical analysis, patients with Gleason score of 8 or more were compared with those with a Gleason score of 7 or less, because patients with a Gleason score of 8 or more had a greater chance of recurrence after radical prostatectomy than those with a Gleason score of 7 or less (31). Similarly, pathological categorization of tumor stage was dichotomized as T3a or less versus T3b or more, because previous studies have demonstrated that patients with pT3b or more tumor stage had a significantly greater chance of recurrence than those having pT3a or less tumor stage (31).

Statistical analysis. The data were analyzed by SSPS version 16.0J software (SSPS Inc., Chicago, IL). Hardy-Weinberg equilibrium analyses were performed to compare the observed and expected genotype frequencies using the $\chi^{2}$ test. The age-adjusted odds ratio (aOR) and $95 \%$ confidence interval (CI) for the relative risk of prostate cancer in each genotype were determined by multiple logistic regression analysis with the inclusion of age. The relationships between tumor stage and AI status, and between the genotype and MMP-1 expression level were analyzed by the $\chi^{2}$ test. A probability of $<0.05$ was required for statistical significance.

\section{Results}

Association of the MMP-1 promoter polymorphism with a risk and progression of prostate cancer. The demographics of the study subjects are shown in Table I. The mean age of male controls and prostate cancer patients was 70.0 7 7.6, $72.0 \pm 8.3$, respectively. The numbers of $1 G / 1 G, 1 G / 2 G$, and 


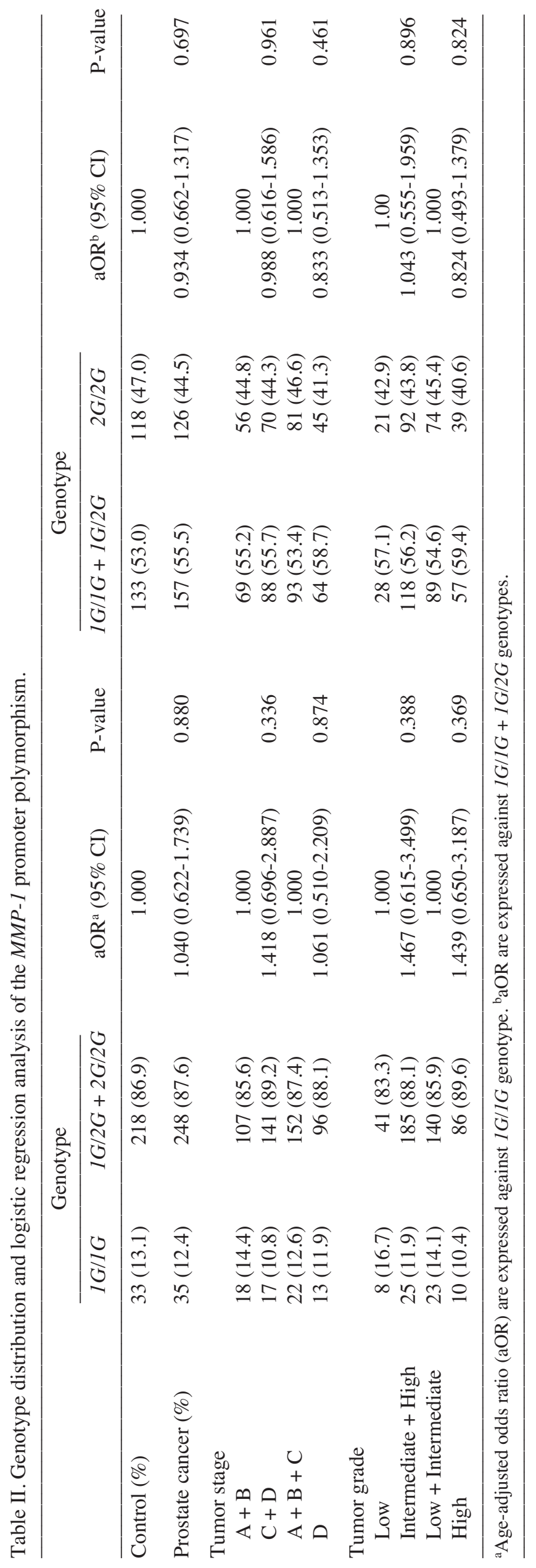

$2 G / 2 G$ genotype in the control group were $33(13.1 \%), 100$ $(39.7 \%)$, and $118(46.8 \%)$, respectively, whereas those in prostate cancer group were $35(12.4 \%), 122(43.1 \%)$, and $126(44.5 \%)$, respectively, demonstrating no statistical significance $(\mathrm{P}=0.746)$. The frequencies of the $1 G$ and $2 G$ in the control group were $166(33.1 \%)$ and $336(66.9 \%)$, whereas those in the prostate cancer group were $192(33.9 \%)$ and 374 $(66.1 \%)$, respectively. There was no statistically significant difference in the allelic frequency between the two groups $(\mathrm{P}=0.768)$. The observed genotype frequency of the polymorphism in the control group did not significantly differ from the expected frequencies according to the Hardy-Weinberg equilibrium (data not shown). The genotype distribution of the $M M P-1$ promoter polymorphism and results of logistic regression analyses are summarized in Table II. Age-adjusted logistic regression analysis showed no significant association between the genotypes and the risk of prostate cancer in either the dominant $(1 G / 1 G$ vs. $1 G / 2 G$ or $2 G / 2 G)$ or the recessive ( $1 G / 1 G$ or $1 G / 2 G$ vs. $2 G / 2 G$ ) models. Next, the associations between the genotypes and the tumor stage were analyzed using both dominant and recessive models. There were no significant differences between stage $\mathrm{A} / \mathrm{B}$ (localized) and stage $\mathrm{C} / \mathrm{D}$ (invasive), stage $\mathrm{A} / \mathrm{B} / \mathrm{C}$ (non-metastatic) and stage $\mathrm{D}$ (metastatic), between low and intermediate/high grade, or between low/intermediate and high grade and low grade cancer.

Association of AI of the MMP-1 promoter region with pathological stage or tumor grade of prostate cancer. Association of AI with pathological T status or tumor grade is summarized in Table III. Of the 77 prostate cancer specimens, an AI was evaluated in 32 informative cases ( $41.6 \%$ of all the cases), of which 13 (40.6\% of informative cases) had an AI. Two $(13.3 \%)$ of the 15 cases with a Gleason score of 7 or less had an AI, whereas $11(64.7 \%)$ of the 17 cases with a Gleason score 8 or more had an AI. Similarly, 7 (29.2\%) of 24 cases with pT3a or less disease had an AI, while $6(75.0 \%)$ of 8 with pT3b or more disease had an AI. Among 13 cases with an AI, retention of $1 G$ and $2 G$ allele was observed in $4(30.8 \%)$ and $9(69.2 \%)$ cases, respectively. The association of the AI status was significant in association with a higher Gleason score $(\mathrm{P}=0.003)$ and a higher pathological tumor stage $(\mathrm{P}=0.022)$. Although a retention allele showed no statistically significant association with a Gleason score $(\mathrm{P}=0.522)$, the frequency of the $2 G$ allele retention significantly increased with tumor invasiveness $(\mathrm{P}=0.026)$ (Table III).

Association of MMP-1 expression with the MMP-1 promoter polymorphism and AI of the MMP-1 promoter region. In 77 patients who underwent radical prostatectomy, we evaluated the relationship between the $M M P-1$ promoter polymorphism and MMP-1 expression levels (Table IV). In immunohistochemical analysis of MMP-1, negative, low, moderate, and high expression was observed in $3(3.9 \%), 14(18.2 \%), 39$ $(50.6 \%)$, and $21(27.3 \%)$ cases, respectively. Tumors with $1 G / 2 G$ and $2 G / 2 G$ genotypes had a significantly higher MMP-1 expression level compared to those with $1 G / 1 G$ genotype $(\mathrm{P}=0.006$ and 0.013 , respectively), whereas there was no significant difference in the expression level between the $1 G / 2 G$ and $2 G / 2 G$ genotypes $(\mathrm{P}=0.581)$. AI status of 

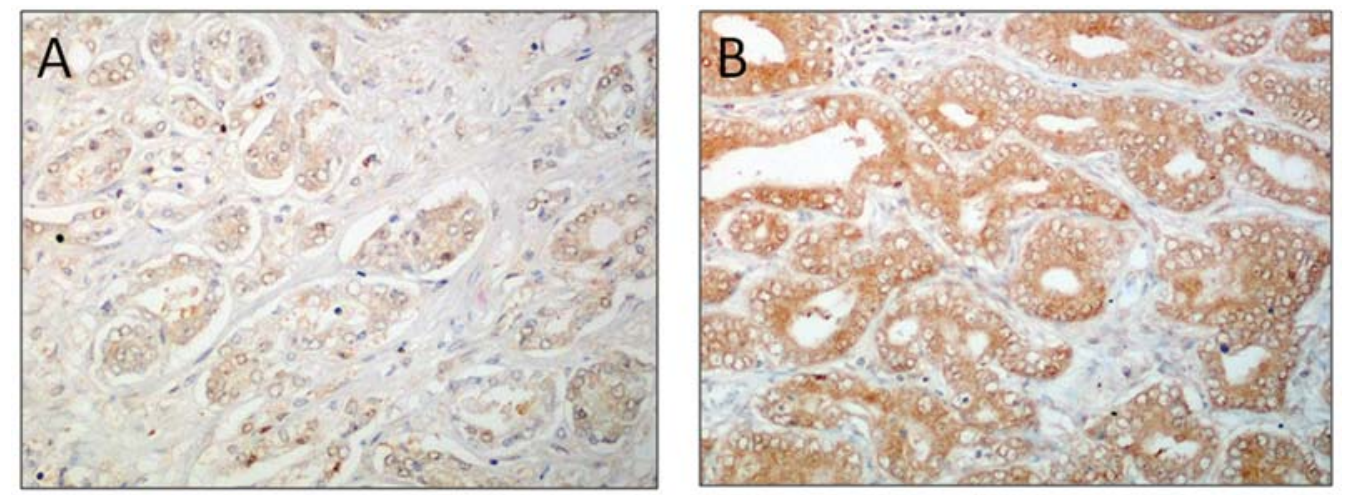

Figure 2. Representative immunohistochemical stainings demonstrating weak (A) and strong (B) expression of MMP-1.

Table III. Association of allelic imbalance and retention allele of the $M M P-1$ promoter region with Gleason score and pathological stage.

\begin{tabular}{|c|c|c|c|c|c|c|c|c|}
\hline & \multirow[b]{2}{*}{ Total } & \multirow[b]{2}{*}{ Informative case } & \multicolumn{2}{|c|}{ Allelic imbalance } & \multirow[b]{2}{*}{ P-value } & \multicolumn{2}{|c|}{ Retention allele } & \multirow[b]{2}{*}{ P-value } \\
\hline & & & - & + & & $1 G$ & $2 G$ & \\
\hline \multicolumn{9}{|c|}{ Gleason score } \\
\hline$\leq 7$ & 44 & 15 & 13 & 2 & & 1 & 1 & \\
\hline$\geq 8$ & 33 & 17 & 6 & 11 & 0.003 & 3 & 8 & 0.561 \\
\hline \multicolumn{9}{|l|}{$\mathrm{T}$ status } \\
\hline$\leq \mathrm{pT} 3 \mathrm{a}$ & 55 & 24 & 17 & 7 & & 4 & 3 & \\
\hline$\geq \mathrm{pT} 3 \mathrm{~b}$ & 22 & 8 & 2 & 6 & 0.022 & 0 & 6 & 0.026 \\
\hline
\end{tabular}

Table IV. Association of MMP-1 expression with the polymorphism and allelic imbalance in the $M M P-1$ promoter region.

\begin{tabular}{llcccc}
\hline & \multicolumn{3}{c}{ Genotype } & & \multicolumn{2}{c}{ Allelic imbalance } \\
\cline { 2 - 4 } $\mathrm{IHC}^{\mathrm{a}}$ & $1 G / 1 G$ & $1 G / 2 G$ & $2 G / 2 G$ & & + \\
\hline Negative & $1(11.0)$ & $1(3.1)$ & $1(2.8)$ & $1(9.1)$ & $0(0.0)$ \\
Weak & $4(44.5)$ & $4(12.5)$ & $6(16.7)$ & $3(18.2)$ & $1(7.6)$ \\
Moderate & $4(44.5)$ & $16(50.0)$ & $19(52.8)$ & $10(45.6)$ & $6(46.2)$ \\
Strong & $0(0.0)$ & $11(34.4)$ & $10(27.8)$ & $5(27.3)$ & $6(46.2)$ \\
P-value & & $0.006^{\mathrm{b}}$ & $0.013^{\mathrm{b}}$ & & 0.156 \\
& & $0.581^{\mathrm{c}}$ & & \\
\hline
\end{tabular}

${ }^{\mathrm{a}} \mathrm{IHC}$, immunohistochemistry; ${ }^{\mathrm{b}} \mathrm{vs} .1 G / 1 G$; ${ }^{\mathrm{v}} \mathrm{vs} .1 G / 2 G$.

tumors was not significantly associated with the MMP-1 expression level ( $\mathrm{P}=0.156)$ (Table IV).

\section{Discussion}

The association of the $2 G$ allele of the polymorphism with a higher MMP-1 expression has been reported in several cancer types $(4,12,21,32)$. Immunohistochemical or gene expression analyses of surgical specimens showed that ovarian, cervical, and endometrial cancers with the $2 G$ allele had a higher expression of MMP-1 than those with the
$1 G / 1 G$ genotype $(4,12,21)$, and tongue cancers with the $2 G / 2 G$ genotype showed a higher MMP-1 expression than those with the $1 G$ allele (32). Our study demonstrated that prostate cancer with the $2 G$ allele also had a significantly higher MMP-1 expression level than that with $1 G / 1 G$ genotype, the observation being consistent with previous studies. The increased expression of MMP-1 under the existence of the $2 G$ allele was confirmed by the luciferase assay using constructs with either $1 G$ or $2 G$ at the promoter region $(-1602 \mathrm{bp})$ in fibroblast, melanoma, and breast cancer cell lines $(10,11)$. In these cells, the $2 G$ construct resulted in a 
2- to 29-fold increase in reporter transcription compared with the $1 G$ construct. In the present study, despite the increased expression of MMP-1 in specimens with the $2 G$ allele, progression of the disease did not seem to be affected by the existence of the $2 G$ allele alone. Since the $11 \mathrm{q} 22$ region contains a cluster of $M M P$ genes including $M M P-1,3,7,8$, $10,12,13,20$, and 27 (33), the amplification of $11 \mathrm{q} 22$ region may induce co-overexpression of other MMPs concurrent with MMP-1 and cooperatively facilitates tumor invasion.

In this study, the tumor progression was significantly associated with an AI, especially retention of the $2 G$ allele of the $M M P-1$ promoter region, whereas our case-control study showed that the presence of the $2 G$ allele alone did not seem to have an effect on the tumor progression. Noll et al also demonstrated that $83 \%$ of metastatic melanoma with an AI of the region had retention of the $2 G$ allele (34). Our study, however, did not reveal whether each AI case had a loss or gain of either allele. A previous study using comparative genomic hybridization (CGH) analyses showed that $11 \mathrm{q} 22$ was one of the most frequently amplified regions in localized prostate cancer (27). In the study, 5 $(22.7 \%)$ of 22 cases with pT2 disease and $6(75.0 \%)$ of 8 with pT3 disease had a gain in 11q22 region, an observation consistent with our findings. The finding that advanced tumors have a disproportionate representation of the $2 G$ allele implies that the retention and probably amplification of the $2 G$ allele has a selective advantage for tumor cells to acquire metastatic or invasive potential. Further studies combined with fluorescence in situ hybridization or CGH analysis are warranted to determine the gene dosage effect of the $2 G$ allele on prostate cancer progression. In renal cell cancer, although $\mathrm{AI}$ of the $11 \mathrm{q} 22$ region was not detected, the $2 G / 2 G$ genotype showed a significant association with cancer susceptibility (17). The contribution of the $2 G$ allele dosage to carcinogenesis or tumor progression varies among different cancer types (17).

There is only one small-scale study regarding prostate cancer, which showed that the $M M P-1$ promoter polymorphism was not associated with susceptibility to the disease (29). Our relatively large-scale study also did not find any association with susceptibility to prostate cancer. Some potential explanations for the lack of association include the fact that $M M P-1$ polymorphism is not involved in carcinogenesis of prostate cancer, but contributes to tumor invasion or progression. Another reason could be that the sample size in this case-control study did not have sufficient statistical power to detect minimal differences in genotype frequency between the control and patient groups. A larger-scale study is therefore needed to validate our results.

It is unclear whether the polymorphism possesses direct effects on the development of malignant tumors or is only a genetic marker predicting susceptibility to cancers. The $M M P-1$ promoter polymorphism is known to be in linkage disequilibrium with the $5 \mathrm{~A} / 6 \mathrm{~A}$ polymorphism in the promoter region of the $M M P-3,(35,36)$ and it is known that spontaneous breast cancer develops in $M M P-3$ transgenic mice (37). Although a previous study indicated that the $2 G$ allele, which creates ETS binding site, induced higher ERK-mediated MMP-1 expression (11), there has been no apparent evidence that higher expression of MMP-1 itself promotes carcinogenesis. Moreover, the amplified 11q22 region harbors several important genes involved in carcinogenesis and/or cancer progression such as other MMPs, BIRC2, and BIRC3. Thus, when we evaluate the effect of the $M M P-1$ polymorphism or AI on the cancer susceptibility or progression, consideration should be given to the relative contribution of linkage disequilibrium between polymorphisms and other genes located in the co-amplified region.

In conclusion, the presence of the $2 G$ allele was associated with higher expression of MMP-1 in prostate cancer tissue and AI of the $M M P-1$ promoter region, specifically in retention of the $2 G$ allele, was suggested to be involved in the progression of prostate cancer. However, the $M M P-1$ polymorphism itself did not influence susceptibility nor progression of the prostate cancer.

\section{Acknowledgements}

This study was supported by Japan Society for Promotion of Science (19390411, 19591832, 19591833, 19659406). We are grateful to Ms. Y. Mitobe and Ms. T. Shibata (Department of Urology, Akita University School of Medicine, Japan) for their excellent technical assistance.

\section{References}

1. Dvorak HF: Tumors: wounds that do not heal. Similarities between tumor stroma generation and wound healing. N Engl J Med 315: 1650-1659, 1986.

2. Pulukuri SM and Rao JS: Matrix metalloproteinase-1 promotes prostate tumor growth and metastasis. Int J Oncol 32: 757-765, 2008.

3. Murray GI, Duncan ME, O'Neil P, Melvin WT and Fothergil JE: Matrix metalloproteinase-1 is associated with poor prognosis in colorectal cancer. Nat Med 2: 461-462, 1996.

4. Kanamori Y, Matsushima M, Minaguchi T, Kobayashi K, Sagae S, Kudo R, Terakawa N and Nakamura Y: Correlation between expression of the matrix metalloproteinase-1 gene in ovarian cancers and an insertion/deletion polymorphism in its promoter region. Cancer Res 59: 4225-4227, 1999.

5. Murray GI, Duncan ME, O'Neil P, McKay JA, Melvin WT and Fothergill JE: Matrix metalloproteinase-1 is associated with poor prognosis in oesophageal cancer. J Pathol 185: 256-261, 1998.

6. Ala-Aho R and Kahari VM: Collagenases in cancer. Biochimie 87: 273-286, 2005.

7. Murray GI, Duncan ME, Arbuckle E, Melvin WT and Fothergill JE: Matrix metalloproteinases and their inhibitors in gastric cancer. Gut 43: 791-797, 1998.

8. Nikkola J, Vihinen P, Vlaykova T, Hahka-Kemppinen M, Kahari VM and Pyrhonen S: High expression levels of collagenase-1 and stromelysin-1 correlate with shorter disease-free survival in human metastatic melanoma. Int J Cancer 97: 432-438, 2002.

9. Pardo A and Selman M: MMP-1: the elder of the family. Int J Biochem Cell Biol 37: 283-288, 2005.

10. Rutter JL, Mitchell TI, Buttice G, Meyers J, Gusella JF, Ozelius LJ and Brinckerhoff CE: A single nucleotide polymorphism in the matrix metalloproteinase-1 promoter creates an Ets binding site and augments transcription. Cancer Res 58: 5321-5325, 1998.

11. Tower GB, Coon CI and Brinckerhoff CE: The 2G single nucleotide polymorphism (SNP) in the MMP-1 promoter contributes to high levels of MMP-1 transcription in MCF7/ADR breast cancer cells. Breast Cancer Res Treat 82: 75$82,2003$.

12. Nishioka Y, Kobayashi K, Sagae S, Ishioka S, Nishikawa A, Matsushima M, Kanamori Y, Minaguchi T, Nakamura Y, Tokino $\mathrm{T}$ and Kudo R: A single nucleotide polymorphism in the matrix metalloproteinase-1 promoter in endometrial carcinomas. Jpn J Cancer Res 91: 612-615, 2000. 
13. Ghilardi G, Biondi ML, Mangoni J, Leviti S, DeMonti M, Guagnellini E and Scorza R: Matrix metalloproteinase-1 promoter polymorphism $1 \mathrm{G} / 2 \mathrm{G}$ is correlated with colorectal cancer invasiveness. Clin Cancer Res 7: 2344-2346, 2001.

14. Zhu Y, Spitz MR, Lei L, Mills GB and Wu X: A single nucleotide polymorphism in the matrix metalloproteinase-1 promoter enhances lung cancer susceptibility. Cancer Res 61: 7825-7829, 2001.

15. Su L, Zhou W, Park S, Wain JC, Lynch TJ, Liu G and Christiani DC: Matrix metalloproteinase-1 promoter polymorphism and lung cancer risk. Cancer Epidemiol Biomarkers Prev 14: 567-570, 2005.

16. Hinoda Y, Okayama N, Takano N, Fujimura K, Suehiro Y, Hamanaka Y, Hazama S, Kitamura Y, Kamatani N and Oka M: Association of functional polymorphisms of matrix metalloproteinase (MMP)-1 and MMP-3 genes with colorectal cancer. Int J Cancer 102: 526-529, 2002.

17. Hirata H, Naito K, Yoshihiro S, Matsuyama H, Suehiro Y and Hinoda Y: A single nucleotide polymorphism in the matrix metalloproteinase-1 promoter is associated with conventional renal cell carcinoma. Int J Cancer 106: 372-374, 2003.

18. Elander N, Soderkvist P and Fransen K: Matrix metalloproteinase (MMP) -1, -2, -3 and -9 promoter polymorphisms in colorectal cancer. Anticancer Res 26: 791-795, 2006.

19. Nishizawa R, Nagata M, Noman AA, Kitamura N, Fujita $H$, Hoshina H, Kubota T, Itagaki M, Shingaki S, Ohnishi M, Kurita H, Katsura K, Saito C, Yoshie H and Takagi R: The $2 \mathrm{G}$ allele of promoter region of matrix metalloproteinase-1 as an essential pre-condition for the early onset of oral squamous cell carcinoma. BMC Cancer 7: 187, 2007.

20. Tasci AI, Tugcu V, Ozbek E, Ozbay B, Simsek A and Koksal V: A single-nucleotide polymorphism in the matrix metalloproteinase- 1 promoter enhances bladder cancer susceptibility. BJU Int 101: 503-507, 2008.

21. Nishioka Y, Sagae S, Nishikawa A, Ishioka S and Kudo R: A relationship between Matrix metalloproteinase-1 (MMP-1) promoter polymorphism and cervical cancer progression. Cancer Lett 200: 49-55, 2003.

22. Matsumura S, Oue N, Kitadai Y, Chayama K, Yoshida K, Yamaguchi Y, Toge T, Imai K, Nakachi K and Yasui W: A single nucleotide polymorphism in the MMP-1 promoter is correlated with histological differentiation of gastric cancer. J Cancer Res Clin Oncol 130: 259-265, 2004.

23. Zinzindohoue F, Lecomte T, Ferraz JM, Houllier AM, Cugnenc PH, Berger A, Blons $\mathrm{H}$ and Laurent-Puig P: Pro-gnostic significance of MMP-1 and MMP-3 functional promoter polymorphisms in colorectal cancer. Clin Cancer Res 11: 594-599, 2005.

24. Carneiro A, Isinger A, Karlsson A, Johansson J, Jonsson G, Bendahl PO, Falkenback D, Halvarsson B and Nilbert M: Prognostic impact of array-based genomic profiles in esophageal squamous cell cancer. BMC Cancer 8: 98, 2008.

25. Lassmann S, Weis R, Makowiec F, Roth J, Danciu M, Hopt U and Werner M: Array CGH identifies distinct DNA copy number profiles of oncogenes and tumor suppressor genes in chromosomal- and microsatellite-unstable sporadic colorectal carcinomas. J Mol Med 85: 293-304, 2007.
26. Narayan G, Bourdon V, Chaganti S, Arias-Pulido H, Nandula SV, Rao PH, Gissmann L, Durst M, Schneider A, Pothuri B, Mansukhani M, Basso K, Chaganti RS and Murty VV: Gene dosage alterations revealed by cDNA microarray analysis in cervical cancer: identification of candidate amplified and overexpressed genes. Genes Chromosomes Cancer 46: 373-384, 2007.

27. Kasahara K, Taguchi T, Yamasaki I, Kamada M and Shuin T: Genetic changes in localized prostate cancer of Japanese patients shown by comparative genomic hybridization. Cancer Genet Cytogenet 159: 84-88, 2005.

28. Cardillo MR, Di Silverio F and Gentile V: Quantitative immunohistochemical and in situ hybridization analysis of metalloproteinases in prostate cancer. Anticancer Res 26: 973-982, 2006.

29. Albayrak S, Canguven O, Goktas C, Aydemir H and Koksal V: Role of MMP-1 $1 \mathrm{G} / 2 \mathrm{G}$ promoter gene polymorphism on the development of prostate cancer in the Turkish population. Urol Int 79: 312-315, 2007

30. Tsuchiya N, Mishina M, Narita S, Kumazawa T, Inoue T, Horikawa Y, Kakinuma H, Yuasa T, Matsuura S, Satoh S, Ogawa O and Habuchi T: Association of XRCC1 gene polymorphisms with the susceptibility and chromosomal aberration of testicular germ cell tumors. Int J Oncol 28: 1217-1223, 2006.

31. D'Amico AV, Whittington R, Malkowicz SB, Schultz D, Schnall M, Tomaszewski JE and Wein A: A multivariate analysis of clinical and pathological factors that predict for prostate specific antigen failure after radical prostatectomy for prostate cancer. J Urol 154: 131-138, 1995.

32. Shimizu Y, Kondo S, Shirai A, Furukawa M and Yoshizaki T: A single nucleotide polymorphism in the matrix metalloproteinase- 1 and interleukin- 8 gene promoter predicts poor prognosis in tongue cancer. Auris Nasus Larynx 35: 381-389, 2008

33. Puente XS, Sanchez LM, Overall CM and Lopez-Otin C: Human and mouse proteases: a comparative genomic approach. Nat Rev Genet 4: 544-558, 2003.

34. Noll WW, Belloni DR, Rutter JL, Storm CA, Schned AR, Titus-Ernstoff L, Ernstoff MS and Brinckerhoff CE: Loss of heterozygosity on chromosome 11q22-23 in melanoma is associated with retention of the insertion polymorphism in the matrix metalloproteinase-1 promoter. Am J Pathol 158: 691-697, 2001

35. Pendas AM, Matilla T, Estivill X and Lopez-Otin C: The human collagenase-3 (CLG3) gene is located on chromosome 11q22.3 clustered to other members of the matrix metalloproteinase gene family. Genomics 26: 615-618, 1995.

36. Ye S, Dhillon S, Turner SJ, Bateman AC, Theaker JM, Pickering RM, Day I and Howell WM: Invasiveness of cutaneous malignant melanoma is influenced by matrix metalloproteinase 1 gene polymorphism. Cancer Res 61: 1296-1298, 2001.

37. Sternlicht MD, Bissell MJ and Werb Z: The matrix metalloproteinase stromelysin-1 acts as a natural mammary tumor promoter. Oncogene 19: 1102-1113, 2000. 